\title{
ABUNDANCIA Y BIOMASA DE LOMBRICES DE TIERRA EN DOS ECOSISTEMAS INTERVENIDOS DEL BOSQUE TRÓPICAL HÚMEDO, BLUEFIELDS
}

\author{
Las lombrices de tierra son el intestino de la tierra
}

Gilbert White, 1789.

Gladys Luna ${ }^{[1]}$

Karla Sequeira ${ }^{[2]}$

Marielo Torrez ${ }^{[3]}$

Esneyder Taleno ${ }^{[4]}$

Ivania Serrano ${ }^{[5]}$

Maricela González $^{[6]}$

\section{Resumen}

Muchos estudios han demostrado que las lombrices de tierra (Anelidae) son el componente más abundante de la macrofauna del suelo y desempeñan funciones ecológicas dentro de él. El objetivo fue evaluar la abundancia y biomasa de lombrices de tierra en dos hábitats del bosque tropical húmedo con diferentes usos de suelo en la ciudad de Bluefields, Región Autónoma Atlántico Sur (RAAS). En cada hábitat se seleccionaron dos micro -hábitats: uno con sombra y otro sin sombra y en cada uno se delimitaron tres subparcelas de 1 pie $^{2}$ cada una, y una profundidad de $10 \mathrm{~cm}$, ubicadas al azar y a una distancia mínima de $15 \mathrm{~m}$ entre sí. Cada muestra de suelo se tamizó y se extrajeron, se midieron y se pesaron los individuos por subparcela. El número de lombrices registradas fue mayor en el hábitat de área reforestada que en el hábitat de bosque, pero ocurrió lo contrario con respecto al tamaño promedio de los individuos. La mayor disponibilidad de materia orgánica en el bosque puede explicar el mayor tamaño de los individuos en este sitio. En aquellos suelos que han sufrido alteraciones antrópicas, como en el campus universitario, las comunidades de lombrices han experimentado cambios extremos denotando así una mala salud; sin embargo, estas comunidades se están recuperando, especialmente en los microhabitats con sombra.

Palabas claves: Bosque tropical, uso del suelo, lombrices de tierra, micro habitats, bosque secundario, predio reforestado.

\footnotetext{
[1] Máster en Recursos Naturales y Desarrollo Rural. Tutora de la investigación. golunabello@yahoo.com

[2] Ingeniera Agroforestal. Tutora de la investigación. Tutora de la investigación. kysc31@yahoo.com

[3] Ingenieras Agroforestales, URACCAN-Bluefields.

[4] Ingenieras Agroforestales, URACCAN-Bluefields.

[5] Ingenieras Agroforestales, URACCAN-Bluefields.

[6] Ingenieras Agroforestales, URACCAN-Bluefields.
} 


\section{Introducción}

Uno de los procesos más importantes en la fertilidad del suelo es la descomposición de la materia orgánica y las lombrices de tierra juegan un papel trascendental dentro de este proceso (Brussard, 1994).

El presente estudio busca determinar la forma en que están distribuidas las poblaciones de lombrices de tierra en dos hábitats diferentes del bosque tropical húmedo en la Región Autónoma Atlántico Sur: un parche de bosque secundario y un área reforestada en el campus de la URACCAN-Bluefields. Ambos sitios han sido sometidos a diferentes intensidades de uso del suelo y la cobertura vegetal es heterogénea, por lo que se seleccionaron dos micro hábitat dentro de cada hábitats para estudiar estos organismos: con sombra y sin ésta.

Según la literatura, las lombrices son organismos sensibles a la temperatura y a la disponibilidad de materia orgánica y los diferentes usos del suelo implican técnicas e insumos diferenciales que pueden afectar la estructura y composición de sus comunidades, por tanto la presencia o ausencia de estos organismos podría indicar el estado de salud de un suelo determinado (Fragoso, 1993).

Con este trabajo se pretende conocer la dinámica de las poblaciones de lombrices y en base a ello, determinar el estado de salud de los suelos en el campus universitario, dar recomendaciones pertinentes para mejorar la calidad de éstos y de esta forma, favorecer el crecimiento de las plantas e incrementar la biodiversidad asociada en este sitio.

\section{Revisión de Literatura}

\section{Generalidades de las lombrices de tierra (Lombricus terrestres)}

Lombriz es el nombre que se aplica a más de 1,00o especies de gusanos pertenecientes a la clase Oligoqueta del filo Anélidos (White, 1789)

Estos organismos son hermafroditas insuficientes, es decir que un individuo tiene ambos sexo, pero necesita aparearse para reproducirse. La unión sexual se realiza por las noches y puede durar hasta 4 horas. Los huevos eclosionan a las dos o tres semanas de puestos y dan a luz entre 2 y 20 lombrices cada uno. Alcanzan la madurez sexual a las 6 hasta 10 semanas. Si son expuestas a bajas temperaturas, los individuos pueden morir, siendo la temperatura ideal entre $16^{\circ} \mathrm{C}$ a $28^{\circ} \mathrm{C}$. En estado adulto llegan a medir entre 9 y $30 \mathrm{~cm}$ de largo y viven de 4 a 5 años. (Hernández et. al. 1996). 


\section{Importancia ecológica}

Por su gran tamaño con respecto a otros organismos del suelo y por la diversidad de funciones que desempeñan, las lombrices de tierra son consideradas como agentes insustituibles en la conservación de la estructura y en la dinámica del suelo al excavar galerías verticales u horizontales mientras ingieren y remueven el suelo (Swift,1994). Según Lee y Foster (1991), la porosidad del suelo se incrementa por esas galerías, ocurre un mejoramiento en el intercambio gaseoso y en la velocidad de la infiltración del agua, la cual aumenta entre dos y diez veces con respecto a suelos similares sin lombrices. Al ser removido y aireado, por la acción de las lombrices de tierra, el suelo se vuelve más fértil.

Las lombrices son capaces de ingerir grandes cantidades de materia celulósicas, llegando a comer hasta el 90 por ciento de su propio peso por día, de esta ingesta excretan entre el 50 y 60 por ciento convertido en un nutriente natural de alta calidad; el lombrihumus.

\section{Materiales y Métodos}

El trabajo se realizó en dos tipos de ecosistemas. El primero es un parche de bosque secundario intervenido y en proceso de regeneración ubicado al norte de la URACCAN - Bluefields y el segundo es un predio reforestado en el campus de esta Universidad. En cada sitio se realizaron muestreos en dos tipos de micro hábitats, con y sin sombra.

En cada hábitat se establecieron tres subparcelas de 1 pie $^{2}$ y en esta área se excavó el suelo a una profundidad de $10 \mathrm{~cm}$. Las subparcelas fueron establecidas a una distancia mínima de $15 \mathrm{~m}$ entre sí. En cada subparcela se recolectaron todas las lombrices, separando con sumo cuidado la tierra y hojarasca de la muestra, para lo cual se utilizó un tamiz fino. Cada individuo fue medido y colocado en un recipiente, después, cada muestra se etiquetó indicando el hábitat y micro hábitat donde se encontró. Todas las muestras fueron colectadas y rotuladas para su posterior pesado. Para el análisis de datos relacionó el número de individuos y el hábitat y micro hábitat muestreados; así como el peso en gramos y el hábitat y micro hábitat. Además se estimo el tamaño promedio, máximo y mínimo de los individuos colectados por hábitat y finalmente se presentan los datos de la presencia de huevos de lombrices por hábitat. 


\section{Resultados y Discusión}

\section{Caracterización de los micro hábitats:}

\section{Bosque intervenido con sombra:}

Las especies vegetales que predominan en el área son Miconia argéntea, Schefflera morototoni, Lacistema agregatum, Vochysia ferruginea, y algunas especies de las familias Melastomataceae, Fabaceae, Dileniaceae, entre otros. El suelo es de tipo arcilloso, con alta humedad y presencia de mucha materia orgánica en descomposición.

Altas cantidades de hojarasca, árboles de 10-15 mts de altura, suelo húmedo con abundantes raíces. El área en general ha sido sometida a despales selectivos constantes (árboles de diferentes tamaños, especies y ubicación) por parte de la población aledaña.

\section{Bosque intervenido sin sombra:}

Suelos con menor humedad relativa, mayor temperatura superficial y entrada de luz solar difusa, rodeado de árboles de 10-15 mts de altura, presencia de raíces y hojarasca. Suelos más pedregosos.

\section{Predio reforestado con sombra:}

El predio reforestado, se encuentra dominado por especies tales como Terminalia catappa, Anacardium occidentalis y Cocus nucifera: son suelos removidos con maquinaria pesada a inicios de los años 9o, caracterizados por una alta presencia de piedras grandes, medianas y pequeñas y material inorgánico, donde la fauna edáfica probablemente es incipiente.

Abundancia de hojarasca en todos los estados de descomposición y mineralización, presencia de raíces grandes, muchas piedras, árboles frutales de 4-6 mts de altura y dominancia de pasto natural. Humedad relativa del suelo media-alta. Entrada de sol durante algún momento del día.

\section{Predio reforestado sin sombra:}

Suelo cubierto de gramas o en parches de grama, muchas piedras grandes, suelo muy seco, ausencia de árboles, algunas raíces finas, se encuentran bajo sol todo el día. Además se encontró evidencia de materia inorgánica. 


\section{Abundancia y densidad de lombrices}

Los resultados indican que existe una diferencia significativa entre el número de individuos (lombrices) encontrados en los dos hábitat, siendo mayor en el área reforestada que en el bosque secundario. En total se encontraron 50 individuos en el hábitat de bosque secundario y 82 individuos en el hábitat reforestado. Sin embargo, es posible ver que en ambos hábitat (bosque y área reforestada), la cantidad de individuos en el micro hábitat bajo sombra fue considerablemente mayor que en el micro hábitat bajo sol. (Fig.1)

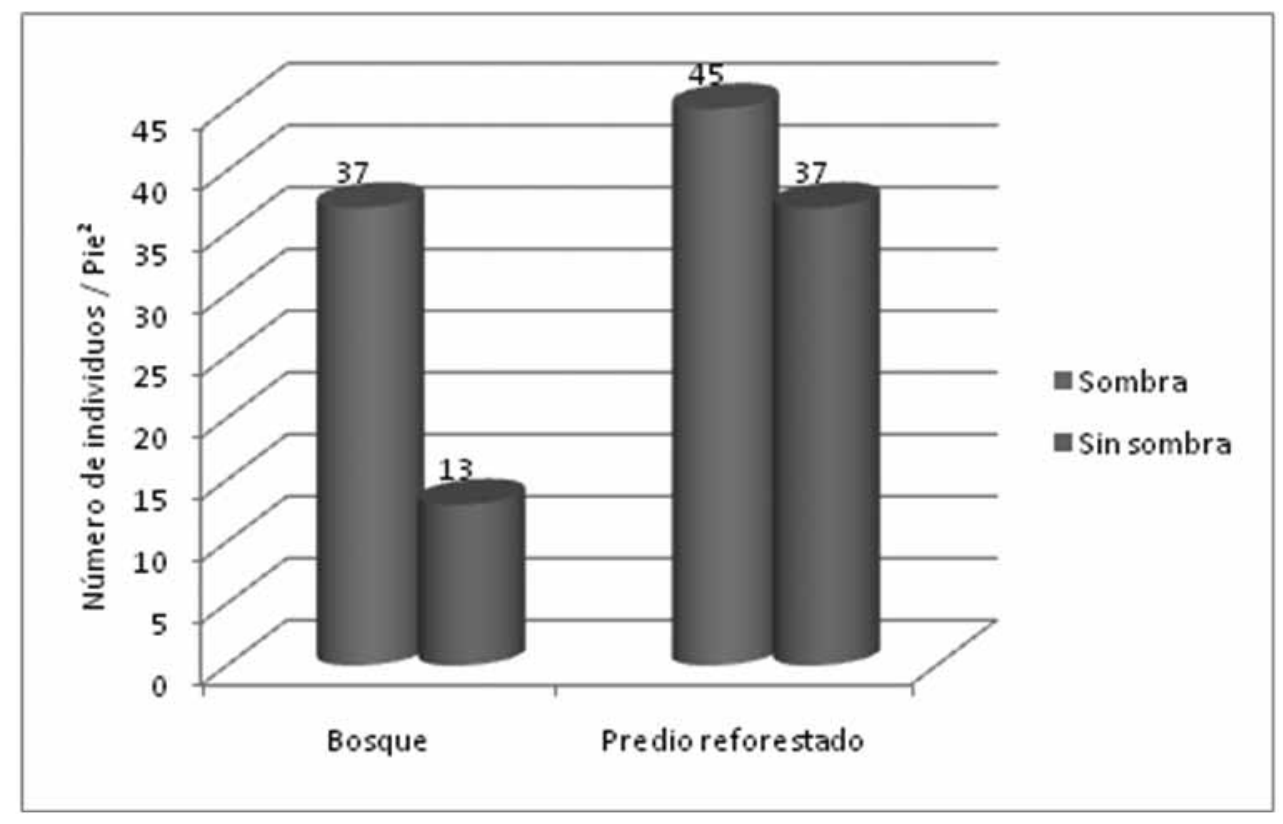

Figura N0. 1. Número de individuos recolectados (pie²) en cada hábitat y micro hábitat estudiado.

Lo que indica que la sombra es un factor clave para el desarrollo de las comunidades de lombrices. Probablemente los sitios con sombra, que están asociados a la presencia de árboles, son los sitios donde hay mayor producción de materia orgánica, lo cual es un requisito para que se desarrolle una comunidad de lombrices, tal y como muestran los resultados. De acuerdo con la literatura, estos organismos suelen vivir en las capas superiores, pero en invierno se entierran más para escapar de las heladas, lo que podría explicar en parte el hecho de haber registrado menor cantidad de lombrices en el bosque, pues en este ecosistema la temperatura suele ser menor debido a la presencia de árboles.

Es interesante el hecho que en todas las muestras del hábitat de bosque las lombrices encontradas fueron de mayor tamaño y grosor con respecto a las del hábitat de área reforestada, las cuales presentaron menor tamaño y grosor. 
Contrario a los resultados encontrados en términos de abundancia entre los dos hábitat muestreados (Fig. No. 1), el hábitat de bosque secundario resultó el sitio donde se encontró el mayor peso de los individuos, mientras que el área reforestada presentó los organismos con un peso mucho menor. En ambos micro hábitats (con y sin sombra) la biomasa viva de los individuos del hábitat bosque presentó mayor peso que en el hábitat reforestado.

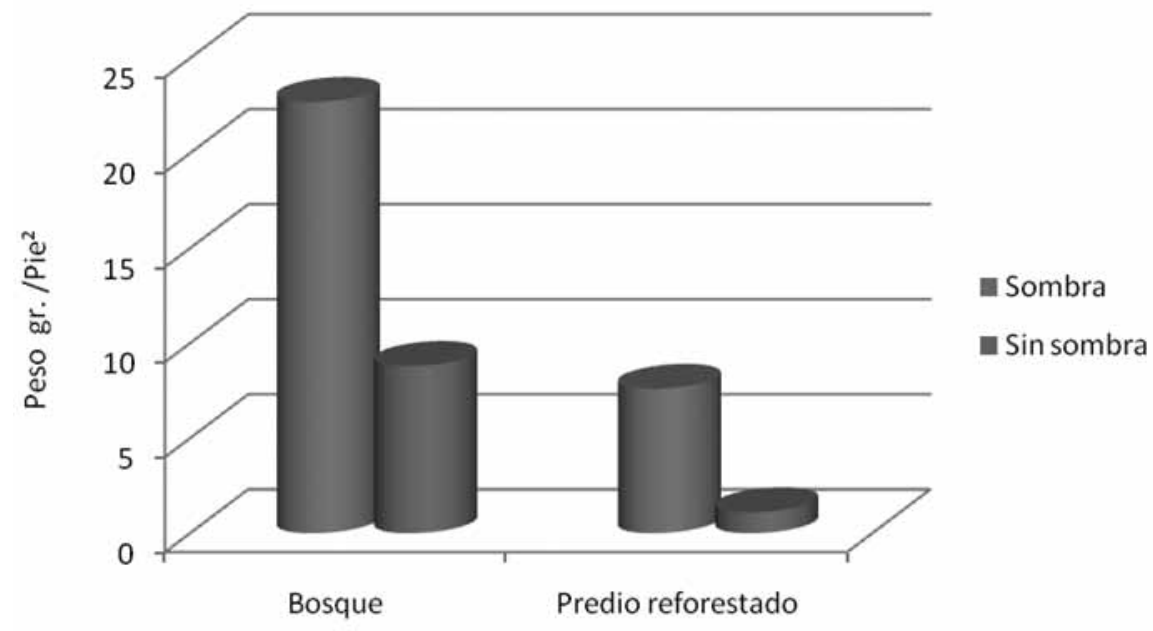

Figura No. 2. Peso total (gramos) por hábitat y micro hábitat.

Resultados que se pueden relacionar con la disponibilidad de nutrientes de la que disponen los individuos en el bosque, pues al haber mayor número de árboles y alta humedad relativa en el ambiente, hay mayor producción de materia que estará disponible (rápida descomposición) para que las comunidades de lombrices puedan crecer, en este caso, en función del tamaño de sus individuos y no necesariamente del número de estos. 


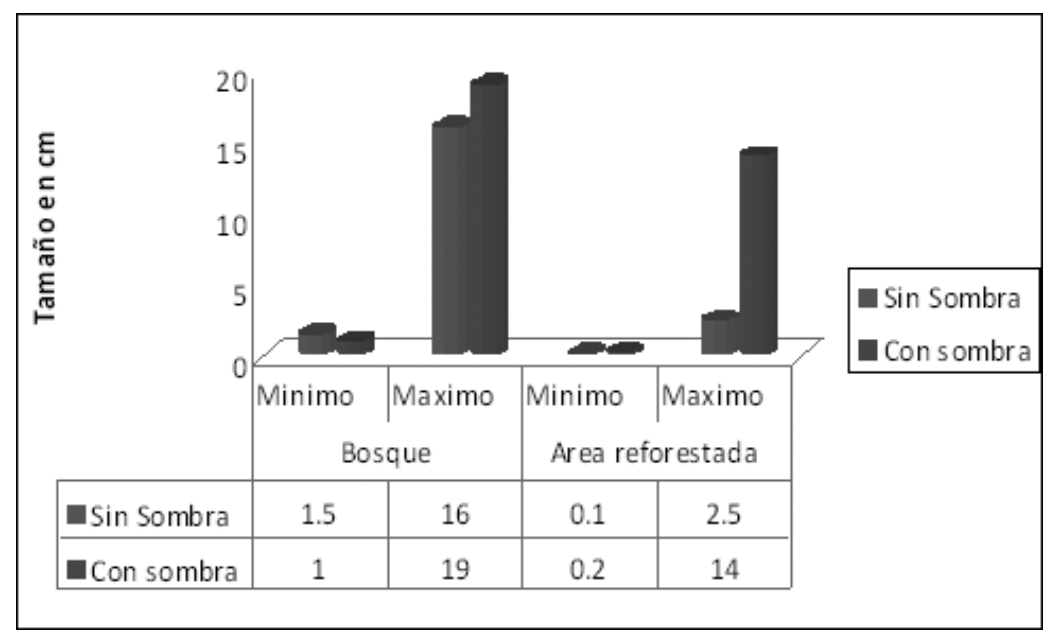

Figura No. 3.Tamaño mínimo y máximo de los individuos encontrados en cada hábitat y micro hábitat.

En la figura No. 3 se observa que el hábitat de bosque presentó los individuos de mayor tamaño, tanto en el microhabitat con sombra $(19 \mathrm{~cm})$ como el de sin sombra $(16 \mathrm{~cm})$, mientras que el tamaño de los individuos del área reforestada fue muy bajo, especialmente en el área sin sombra $(2.5 \mathrm{~cm})$. A pesar de los resultados generales, es importante resaltar que en el microhabitat con sombra del hábitat área reforestada el tamaño máximo de los individuos $(14 \mathrm{~cm})$ es similar a los resultados de ambos microhabitat del hábitat de bosque, por lo que se reafirma lo antes expuesto, que la sombra es un factor que influye directamente en el tamaño de estos organismos. Sin embargo, el tamaño mínimo en el hábitat área reforestada es considerablemente menor $(0.1 \mathrm{~cm}$ y $0.2 \mathrm{~cm}$ ) que en el hábitat de bosque, donde el organismo más pequeño registrado es diez veces mayor que en el microhabitat área reforestada.

Esto podría indicar que las lombrices de tierra utilizan diferentes estrategias dependiendo del hábitat donde se encuentran. Probablemente en el hábitat del bosque ponen más energía en producir individuos más grandes y saludables que en tener muchos, mientras que en el área reforestada, la estrategia es producir muchos individuos, aunque estos sean pequeños. Dato que se corresponde con la cantidad de huevos encontrados en ambos hábitats. 


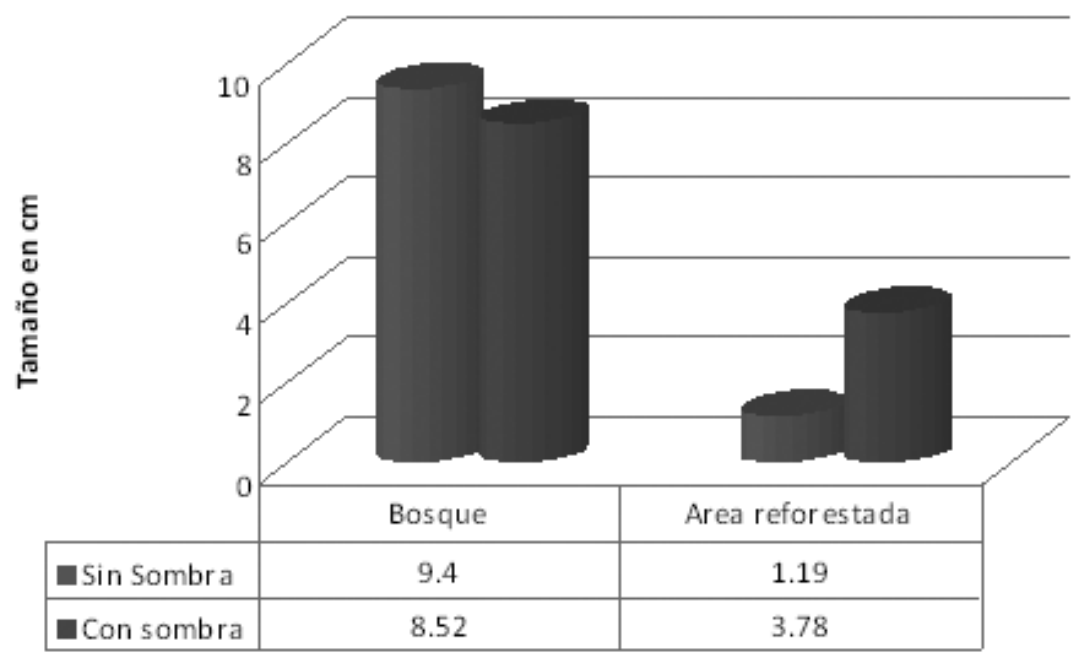

Figura No. 4. Tamaño promedio de los individuos de lombrices en dos hábitats y micro hábitat.

\section{Presencia de huevos por hábitat y micro hábitat}

Los datos indican que existe una pequeña diferencia en el número de huevos encontrados en cada hábitat, siendo mayor el número de huevos en el hábitat reforestado (8 huevos total) con respecto al hábitat bosque, donde solamente se encontraron 5 huevos. Sin embrago, es importante notar que en ambos hábitat se encontraron huevos de lombrices únicamente en los micro hábitat con sombra, mientras que en los sitios sin sombra la presencia de huevos fue nula (Fig.3).

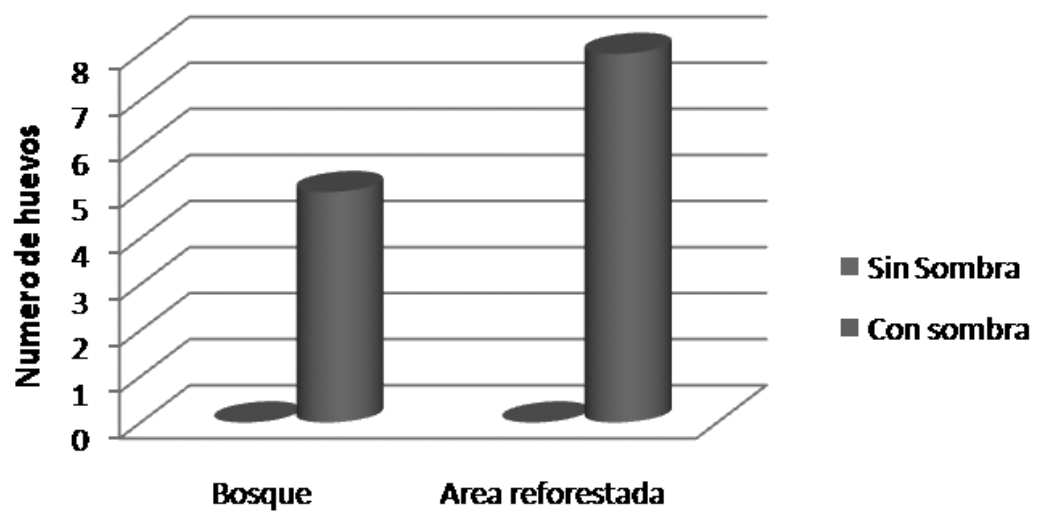

Habitat

Figura No. 5. Presencia/ausencia de huevos de lombrices de tierra en cada hábitat y micro hábitat estudiados. 
Probablemente, estos organismos necesiten sitios frescos y húmedos para que sus huevos puedan eclosionar, lo que podría explicar la mayor abundancia de huevos en los microhabitat con sombra.

La producción y acumulación de lombrices en la superficie del suelo tiene efectos benéficos en la formación de suelo y en el aporte de materia orgánica, como en el caso del bosque y del predio reforestado, por lo tanto es importante conservar y/o incrementar estas áreas dentro del campus de la universidad y en el parche de bosque secundario, para que las lombrices puedan reproducirse y eventualmente contribuir a mejorar la calidad de estos suelos.

\section{Consideraciones}

Las razones por la que se encontró menor número de individuos en el hábitat del bosque pueden ser diversas, a continuación algunos argumentos:

- Las subparcelas probablemente fueron excavadas a muy poca profundidad, ya que según algunos autores cuando el clima es muy caluroso, las lombrices se entierran más para evitar la deshidratación.

- Los muestreos se realizaron de día y según la literatura, las lombrices de tierra rehúyen de la luz del día y salen a la superficie durante la noche para alimentarse y expulsar el detritus. Por tanto el método de muestreo así como la hora pudieron influir en los resultados.

\section{Conclusiones}

En suelos que han sufrido alteraciones, como el caso del área reforestada de la URACCAN, las comunidades de lombrices han experimentado cambios extremos; sin embargo, estas comunidades aparentemente se están recuperando, especialmente en sitios con sombra.

Aunque el número de lombrices registradas fue mayor en el hábitat de área reforestada que en el hábitat de bosque, ocurrió lo contrario con respecto al tamaño promedio de los individuos, lo cual podría ser explicado por la mayor disponibilidad de materia orgánica que existe en el bosque. Esto podría indicar además, que estos organismos utilizan diferentes estrategias dependiendo del hábitat donde se encuentran. La presencia de huevos en ambos hábitat muestreados está directamente relacionados con la presencia de sombra. 
Es necesario conservar y/o incrementar las áreas reforestadas dentro del campus de la universidad y en el parche de bosque secundario, para que las lombrices puedan reproducirse y eventualmente contribuir a mejorar la calidad de estos suelos.

\section{Recomendaciones}

Es necesario promover la reforestación en el campus de la URACCAN para ayudar a incrementar la abundancia de lombrices de tierra y consecuentemente mejorar la calidad de los suelos.

Se debe conservar y proteger el fragmento de bosque ubicado al norte de la universidad; ya que este es el hábitat de estos importantes organismos de suelo, pero a la vez es un laboratorio para los estudiantes de la carrera de ingeniería agroforestal para seguir realizando este tipo de investigaciones.

\section{Lista de Referencias}

Brussaard, L. (1994). Interrelationships between biological activities, soil properties and soil management. In D. J. Greeland and Szabolcs (Eds). Soil resilance and sustainable land use, pp 235-247. CAB International. UK.

Fragoso, G. C. (2002). Las lombrices de tierra en México: diversidad, distribución y manejo. II Simposium Internacional y Reunión Nacional Lombricultura y abonos orgánicos.

Hernández J., Montes de Oca P. \& Villalobos, C. (1996). Comportamiento de la Lombriz de Tierra Eisenia Foetida en seis Sustratos a Base de Estiércoles y Leguminosas. Escuela de Ciencias Agrarias, Universidad Nacional. Resumen X Congreso Nacional Agronómico III Congreso de Suelos.

Jiménez Jaén, Juan José. (2003). Estructura de las comunidades y dinámica de las poblaciones de lombrices de tierra en las sabanas naturales y perturbadas de Carimagua, Colombia. Tesis Doctoral.

Swift, M. J., L. Bohre, S. E. Carter, A. M. Izac and P. L. Woomer. 1994. Biological management of tropical soils: intregrating processes research and farm practice. In $\mathrm{P}$. L. Woomer and M. J. Swift (Eds). The biological management of tropical soil fertility. pp 209-229. John Wiley \& Sons. UK. 